\title{
MODELLING 3D STEAM TURBINE FLOW USING THERMODYNAMIC PROPERTIES OF STEAM IAPWS-95
}

\author{
A.V. Rusanow ${ }^{1}$ \\ P. Lampart ${ }^{2}$ \\ N.V. Pashchenko \\ R.A. Rusanov ${ }^{2}$ \\ ${ }^{1}$ The Podgorny Institute of Mechanical Engineering Problems \\ National Academy of Science of Ukraine in Kharkov, Ukraine \\ ${ }^{2}$ The Szewalski Institute of Fluid Flow Machinery, Polish Academy of Sciences in Gdańsk, Poland
}

\begin{abstract}
An approach to approximate equations of state for water and steam (IAPWS-95) for the calculation of three-dimensional flows of steam in turbomachinery in a range of operation of the present and future steam turbines is described. Test calculations of three-dimensional viscous flow in an LP steam turbine using various equations of state (perfect gas, Van der Waals equation, equation of state for water and steam IAPWS-95) are made. The comparison of numerical results with experimental data is also presented.
\end{abstract}

Keywords: Three-dimensional viscous flow, steam turbine, low-pressure cylinder, sets of equations of state for water and steam IAPWS-95, approximations of equations

\section{INTRODUCTION}

Throughout the last 10-15 years, the methods of viscous flow modeling based on Reynolds equations [1, 2, 3] have been widely applied in calculation, design and optimization of turbomachinery parts, including steam turbines. Adopting such approaches has made a significant improvement in gas dynamic efficiency of turbomachinery, especially with the use of 3D modeling of blade channels, including blade lean, sweep, end wall contouring, radial-axial stages. There are still a number of difficulties in numerical integration of the set of Reynolds equations related to the accuracy and stability of different schemes, formulation of boundary conditions, closing equations (turbulence modeling, equation of state etc.). Most of the application packages use rather simple state equations such as the equation of state for perfect gas, Tammann or Van der Waals equations. In many cases their use is justified and appropriate. However, in relation to the processes in which a phase transition in the working medium occurs (for example in low-pressure cylinders and condensers of conventional, nuclear and marine steam turbines), the use of simple state equations allows modeling of flow structures and rough assessment of kinetic energy losses, but may produce significant uncertainties in the determination of quantities like temperature, mass flow rate and turbine power. In order to describe thermodynamic properties of water and steam using simpler computation methods (one-dimensional, quasisymmetric), one can implement state equations based on the Mayer and Bogomolov equation [4] whose viral coefficients are found by correlation with the experiment. At present, two international standards of state equations for water and steam are used: IAPWS-95 formula [5] for scientific calculations and its simplified version - IAPWS-IF97 formula [6] for industrial calculations. It is difficult to use these state equations in three-dimensional calculations on present-day computers since they increase the required computational time by 1 to 2 orders of magnitude. The most frequently used state equation nowadays in three-dimensional models is the equation with two terms of compressibility coefficient.

This paper presents an approach to the approximation of state equations IAPWS-95 with the use of cubic curves 
for the given arrays of thermodynamic functions. The idea of a similar approximation was first presented in [7]. This method assures a sufficient accuracy of determination of thermodynamic quantities within the whole range of operation of steam turbines (the maximum error does not exceed $\pm 0,1 \%$ ). In the paper, three-dimensional calculations of flow in LP turbines using numerous equations of state are presented and compared with the available experimental data.

\section{METHODS FOR THE CALCULATION OF THREE DIMENSIONAL VISCOUS FLOW IN TURBINES}

The calculation is carried out using the application package FlowER [3] and is based on the integration of the Reynolds equation. In order to determine turbulent effects, the Menter SST model is used [8]. The governing equations are solved with the use of implicit Godunov-type ENO scheme [9]. In order to close the Reynolds equations, equations of state for a perfect gas [10], Tammann equations [11] or Van der Walls equations [4] with constant or variable specific heats are available in the application package FlowER. A number of thermodynamic dependencies, which shape depends on the used state equation, are applied in the process of computation and post processing of results such as:

$$
\begin{aligned}
& T=T(p, \rho) \text { - temperature; } \\
& \rho=\rho(p, T)-\text { density; } \\
& \varepsilon=\varepsilon(p, \rho) \text { - internal energy; } \\
& p=p(\varepsilon, \rho) \text { - pressure; } \\
& a=a(p, \rho) \text { - speed of sound; } \\
& i=i(p, \rho)-\text { enthalpy; } \\
& c_{v}=c_{v}(p, \rho) \text { - specific isochoric heat; } \\
& c_{p}=c_{p}(p, \rho) \text { - specific isobaric heat; }
\end{aligned}
$$$$
S=S(p, \rho) \text { - entropy; }
$$$$
\left(\frac{\partial \varepsilon}{\partial p}\right)_{\rho}=\varepsilon_{p}(p, \rho) \begin{aligned}
& \text { - partial derivative of internal energy } \\
& \text { with respect to pressure at a constant } \\
& \text { density; }
\end{aligned}
$$$$
\left(\frac{\partial \varepsilon}{\partial \rho}\right)_{p}=\varepsilon_{\rho}(p, \rho) \quad \begin{aligned}
& \text { - partial derivative of internal energy } \\
& \text { with respect to density at a constant }
\end{aligned}
$$
pressure;

$$
\left(\frac{\partial S}{\partial p}\right)_{\rho}=S_{p}(p, \rho) \quad \begin{aligned}
& - \text { partial derivative of entropy with } \\
& \text { respect to pressure at a constant } \\
& \text { density; }
\end{aligned}
$$

$$
\begin{aligned}
& \left(\frac{\partial S}{\partial \rho}\right)_{\rho}=S_{\rho}(p, \rho) \begin{array}{l}
\text { - partial derivative of entropy with } \\
\text { respect to density at a constant } \\
\text { pressure; }
\end{array} \\
& \left(\frac{\partial T}{\partial p}\right)_{\rho}=T_{p}(p, \rho) \begin{array}{l}
\begin{array}{l}
\text { - partial derivative of temperature } \\
\text { with respect to pressure at a constant } \\
\text { density; }
\end{array} \\
\left(\frac{\partial T}{\partial \rho}\right)_{\rho}=T_{\rho}(p, \rho) \begin{array}{l}
\text { - partial derivative of temperature } \\
\text { with respect to density at a constant } \\
\text { pressure; }
\end{array}
\end{array}
\end{aligned}
$$$$
i_{*}=i_{*}(p, \rho, C)-\text { total enthalpy; }
$$$$
T_{*}=T_{*}(p, \rho, C)-\text { total temperature; }
$$$$
p_{*}=p_{*}(p, \rho, C)-\text { total pressure; }
$$$$
\rho_{*}=\rho_{*}(p, \rho, C)-\text { total density; }
$$$$
\rho_{i z}=\rho_{i z}(S, p)-\text { isentropic density; }
$$$$
i_{i z}=i_{i z}(S, p)-\text { isentropic pressure, }
$$

where C - speed. Closing thermodynamic functions for perfect gas, Tammann and Van der Walls equations of state are presented in [12].

\section{APPROXIMATION OF CLOSING THERMODYNAMIC FUNCTIONS ON THE BASIS OF THE EQUATIONS FOR THERMODYNAMIC PROPERTIES OF WATER AND STEAM IAPWS-95.}

Equations of International Association for Properties of Water and Steam IAPWS-95 were formulated in 1995. They were determined on the basis of the fundamental Helmholtz equation $f(\rho, T)$ and can be a combination of polynomials of up to 56 elements [5]. In most cases, it is impossible to find an analytical formula for thermodynamic functions necessary for closing the Reynolds equation. This makes the direct application of IAPWS-95 for modeling threedimensional flow ineffective in regards of computational cost. In an approach presented in [7] the closing functions are determined by interpolation of the already calculated arrays (tables) of base points of thermodynamic quantities. However, due to a significant nonlinearity of thermodynamic functions, storage of large arrays might be necessary in order to assure the acceptable accuracy in defining thermodynamic parameters in a wide range of variation of flow parameters (especially in the range of operating modes of steam turbines).

Some hints how to decrease the dimensions of the stored arrays without a loss to the precision of interpolation are described below. In the case where the independent variables for determination of the thermodynamic values are pressure and/or density, it is justified to consider the pressure and density in a logarithmic scale as independent variables. This, for example, allows us to calculate 3D flow in the HP and LP cylinders of the steam turbine where the pressure changes 
from $3 \cdot 10^{3}$ to $2 \cdot 10^{5} \mathrm{~Pa}$ in the LP cylinder and from $3 \cdot 10^{6}$ to $2,4 \cdot 10^{7} \mathrm{~Pa}$ in the HP cylinder.

Another idea to reduce computational costs and still assure the acceptable accuracy of interpolation of IAPWS-95 equations is to rewrite the equations of state for a perfect gas with dimensionless compressibility coefficients depending on two independent variables, especially $p$ and $\rho$. Range variations of these coefficients are much lower when compared with variations of the sought thermodynamic functions. Therefore it is justified to store the arrays of values of the dimensionless compressibility coefficients instead of values of the sought thermodynamic functions. The closing thermodynamic functions are then derived from analytical relations:

$$
\begin{aligned}
& T=\frac{p}{\rho R z_{-} t(\rho, p)} ; \rho=\frac{p}{i} z_{-} \rho(i, p) ; \varepsilon=\frac{p}{s} \frac{z_{-} \varepsilon(\rho, p)}{z_{-} t(\rho, p)} ; \\
& p=\rho \cdot \varepsilon \cdot z_{-} p(\rho, \varepsilon) ; a=\sqrt{\frac{p}{s} \frac{z_{-} a(\rho, p)}{z_{-} t(\rho, p)}} ; i=\frac{p}{\rho}\left(1+\frac{z_{-} \varepsilon(\rho, p)}{z_{-} t(\rho, p)}\right) ; \\
& C_{v}=R \cdot z_{-} C_{v}(\rho, p) ; C_{p}=R \cdot z_{-} C_{p}(\rho, p) ; S=R \cdot z_{-} S(\rho, p) ; \\
& \varepsilon_{p}=\frac{z_{-} \varepsilon(\rho, p)}{\rho \cdot z_{-} t(\rho, p)} ; \varepsilon_{\rho}=-\frac{p}{\rho} \frac{z_{-} \varepsilon(\rho, p)}{z_{-} t(\rho, p)} ; \\
& S_{p}=\frac{C_{v}}{p} ; S_{p}=-\frac{C_{p}}{\rho} ; T_{p}=\frac{1}{\rho R \cdot z_{-} t(\rho, p)} ; T_{\rho}=-\frac{p}{\rho^{2} R \cdot z_{-} t(\rho, p)},
\end{aligned}
$$

with the use of cubic polynomial curves. The entire iteration method is described in detail in [12].

\section{EVALUATION OF APPROXIMATION ERROR FOR CLOSING THERMODYNAMIC FUNCTIONS.}

For the approximation of dimensionless compressibility coefficients and closing thermodynamic functions of two independent variables, two-dimensional arrays of base points $(4001 \times 2001)$ are used. A solid line in Fig. 1 shows the area for the evaluation of arrays of independent variables - pressure and density:

$500 \mathrm{~Pa}<p<3,5 \cdot 10^{7} \mathrm{~Pa} ; 4 \cdot 10^{-3} \mathrm{~kg} / \mathrm{m}^{3}<\rho<210 \mathrm{~kg} / \mathrm{m}^{3}$.

In the same picture, a dashed line surrounds the working area of steam turbines. Relative approximation errors (an average error based on 1000 points randomly selected from the considered area, or a maximum error) in defining thermodynamic functions were calculated as relative deviations of the value found from the proposed approximation from the value obtained with the help of IAPWS-95 tables:

$$
\begin{gathered}
\xi_{\text {mean }}=\text { mean }\left|\frac{f_{\text {approx }}-f_{I A P W S}}{f_{I A P W S}}\right|, \\
\xi_{\text {max }}=\max \left|\frac{f_{\text {approx }}-f_{I A P W S}}{f_{I A P W S}}\right|
\end{gathered}
$$

The average approximation error for all the calculations does not exceed $0,05 \%$, the maximum error - $0,8 \%$. Within where - gas constant [5], $z_{-} t(\rho, p), z_{-} \rho(i, p), z \varepsilon(\rho, p)$, $z_{-} p(\rho, \varepsilon), z_{-} C_{v}(\rho, p), z_{-} C_{p}(\rho, p), z_{-} S(\rho, p)-$ interpolated dimensionless compressibility coefficients for the corresponding thermodynamic functions. While calculating the density, the independent variable $T$ is replaced with the enthalpy, which facilitates the calculation in the two-phase flow regions. The formulas used to define the partial derivatives of internal energy, entropy, and temperatures are simplified, defined based on the assumption, that the compressibility coefficients remain constants. This simplification also increases the stability of numerical convergence of iterative processes for solving nonlinear equations. Values of dimensionless compressibility coefficients in base points are as follows:

$$
\begin{aligned}
& z_{-} t=\frac{p}{\rho R T} ; z_{-} \varepsilon=\frac{\varepsilon}{R T} ; \\
& z_{-} \rho=\frac{i \rho}{p} ; z_{-} p=\frac{p}{\rho \cdot \varepsilon} ; \\
& z_{-} a=\frac{a^{2}}{R T} ; z_{-} C_{v}=\frac{C_{v}}{R} ; \\
& z_{-} C_{p}=\frac{C_{p}}{R} ; z_{-} S=\frac{S}{R},
\end{aligned}
$$

the working area of steam turbines, the average approximation error does not exceed 0,01\%, maximum - 0,1\%, with the largest errors appearing in the region of low pressure and wet steam.

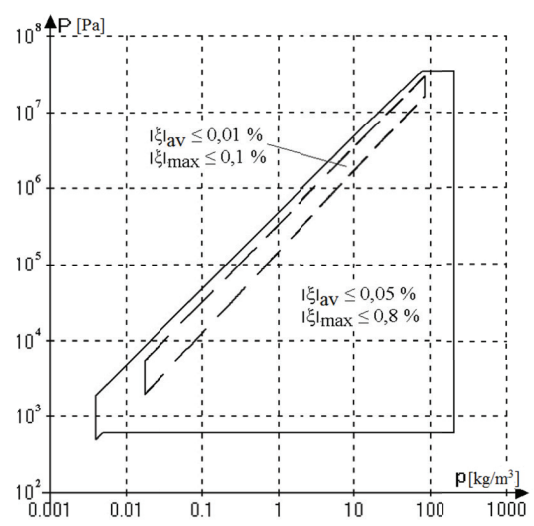

Fig. 1. Working area for approximation of steam properties

\section{COMPUTATIONAL RESULTS}

The presented method of calculation of 3D flow was tested for the case of a low pressure cylinder of a five-stage steam turbine of $360 \mathrm{MW}$ (Fig. 2). The obtained results were compared with the available experimental data [13].

Radial guide vanes are placed at the inlet to the turbine. The stator and rotor blades in all but one stage are shrouded where the corresponding values $p, \rho, T, \varepsilon, i, a, C_{p}, C_{v}$ and are calculated with the use of IAPWS-95 formulations [5]. The interpolation of the compressibility coefficient is determined 
with shrouds equipped with typical labyrinth seals. The rotor blades in the last stage are free-tip. There are two extraction points downstream of stage 3 and 4 . The scheme of regenerative extractions, tip-leakage and labyrinth seal flows in meridional view is presented in Fig. 2.

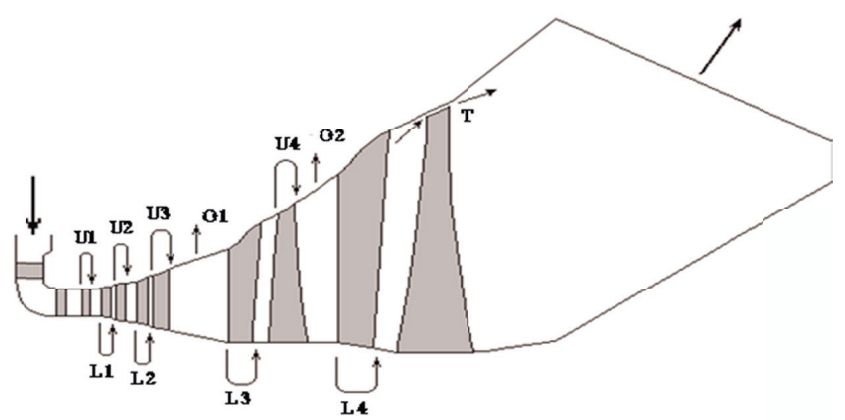

Fig. 2. Meridional cross section of the low pressure flow part of a 360 MW steam turbine. The scheme of leakage and extraction flows, O1, O2 regenerative extractions, $T$ - tip leakage clearance over unshrouded rotor blades, U1-U4 - labyrinth seal slots at the upper contour, L1-L4-labyrinth seal slots at the lower contour.

In order to define boundary conditions for the inlet and outlet of the computational domain, experimental data obtained in [13] are used:

- total pressure at the inlet - $519 \mathrm{kPa}$, total temperature at the inlet - $539 \mathrm{~K}$;

- $\quad$ static pressure at the outlet - $6,2 \mathrm{kPa}$.

The initial data for modeling regenerative extractions and labyrinth seal flows were determined earlier in [14] using a one-dimensional approach. They are included in Table 1 . The flow directions in the labyrinth seals are nominal and are also indicated in Fig. 2. The calculations are made on a structural H-type grid of about 4 million cells in total refined in the boundary layers, according to the assumed turbulence model.

The integral characteristics of flow in the third, fourth and fifth stage of the turbine are calculated first with the use of the equation of state for a perfect gas with variable specific heats (calculation 1), second with the use of the Van der Waals equation of state (calculation 2) and third with the use of the presented method of approximation of IAPWS-95 properties (calculation 3). Figs. 3 and 4 show the comparison of experimental and calculated total and static pressure as well as pitch and swirl angle downstream of stage 3, 4 and 5 .

a)

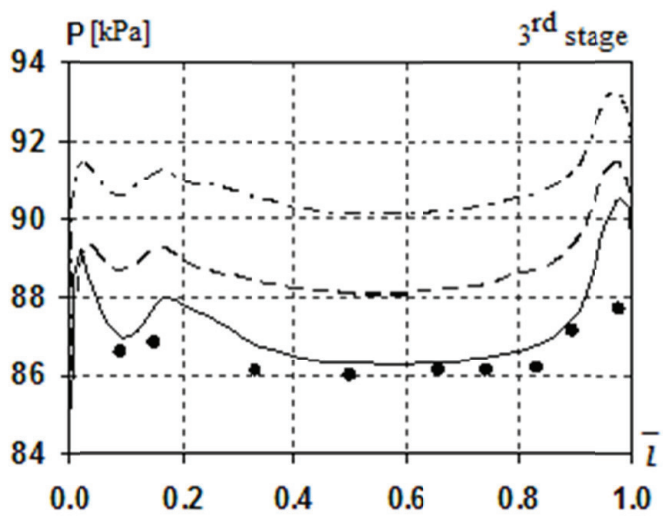

b)

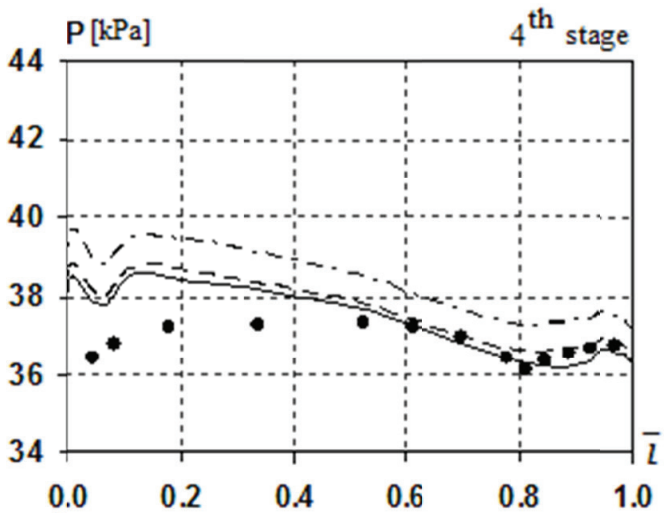

c)

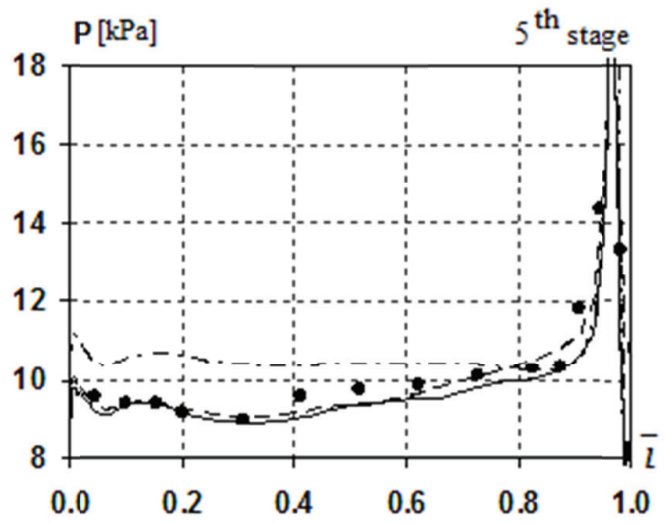

Table 1. Initial data for modeling regenerative extractions and labyrinth seal flows

\begin{tabular}{|c|c|c|c|c|}
\hline Position & Stage & Blade row & $\mathrm{G}, \mathrm{kg} / \mathrm{c}$ & $\mathrm{T}_{0} *, \mathrm{~K}$ \\
\hline U1 & 1 & rotor 1 & 2,00 & 537,8 \\
\hline $\mathrm{L} 1$ & 2 & stator 2 & 1,90 & 487,8 \\
\hline U2 & 2 & rotor 2 & 1,40 & 487,8 \\
\hline $\mathrm{L} 2$ & 3 & stator 3 & 1,21 & 437,8 \\
\hline U3 & 3 & rotor 3 & 1,10 & 437,8 \\
\hline 01 & $3-4$ & - & 5,50 & - \\
\hline L3 & 4 & stator 4 & 0,54 & 387,8 \\
\hline U4 & 4 & rotor 4 & 1,10 & 387,8 \\
\hline $\mathrm{O} 2$ & $4-5$ & - & 5,00 & - \\
\hline L4 & 5 & stator 5 & 0,36 & 337,8 \\
\hline
\end{tabular}


d)

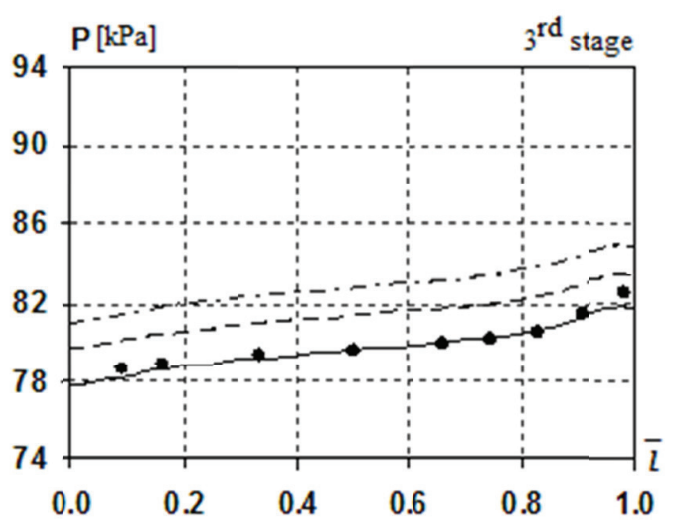

e)

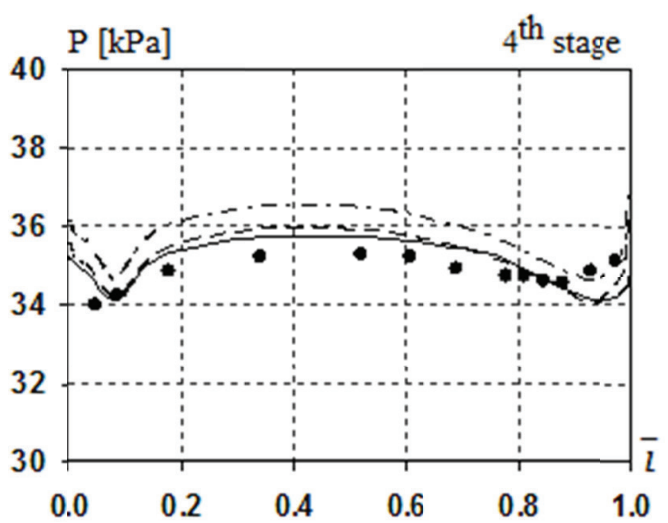

f)

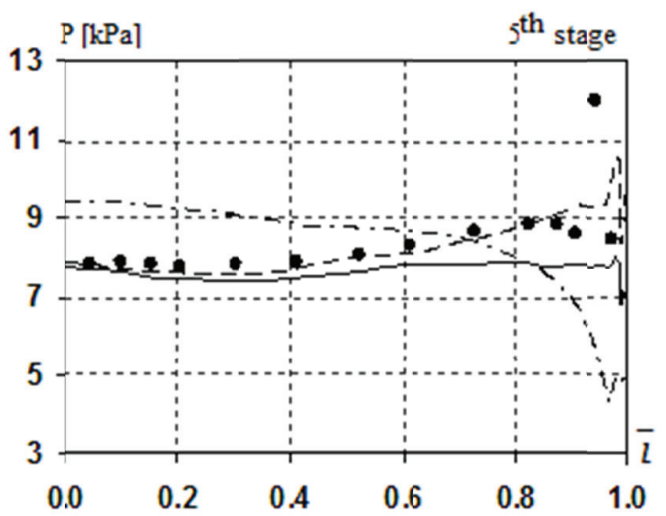

Fig. 3. Total pressure $(a, b, c)$ and static pressure $(d, e, f)$ distribution along the channel height in stage 3,4 and 5;

- experiment, -- --- calculation 1,-- -- -- calculation 2, -----c calculation 3

The presented examples prove consistency of the experimental and calculated data. It is clear that, the best fit belongs to calculation 3 , that is to the method of approximation of IAPWS-95 properties. It should also be noted, that constants in the equations of state used for calculations 1 and 2 were found from experimental data, while it is not necessary to use these data for calculation 3 . a)

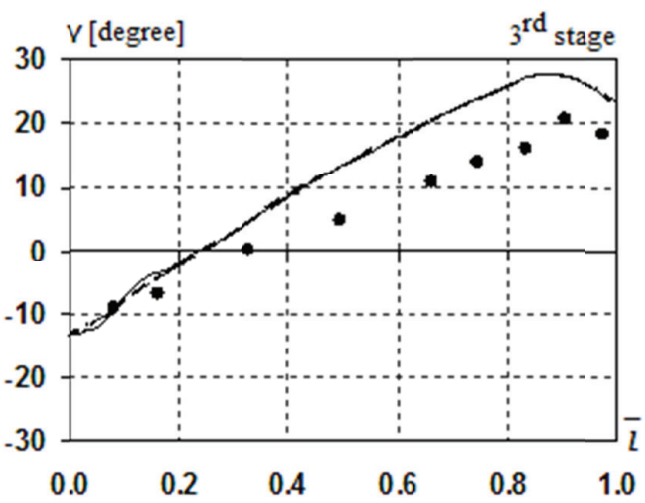

b)

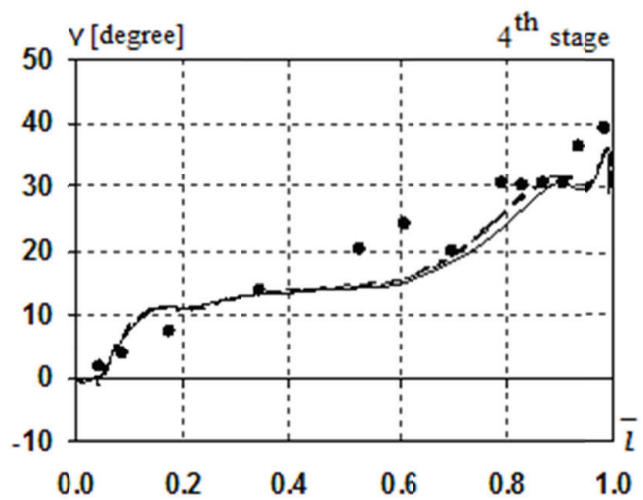

c)

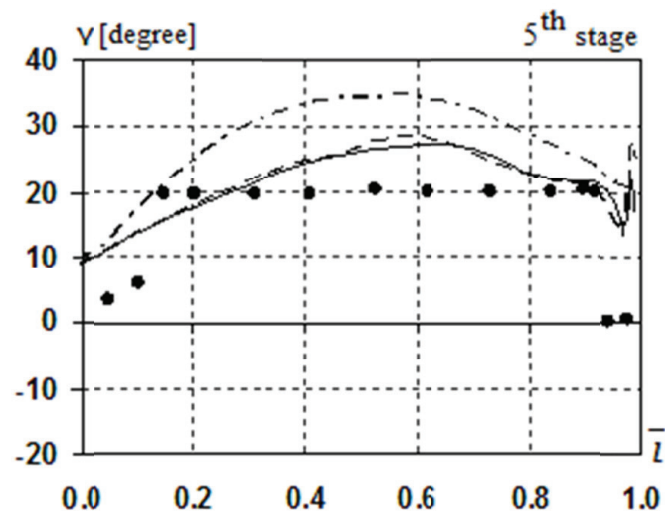

d)

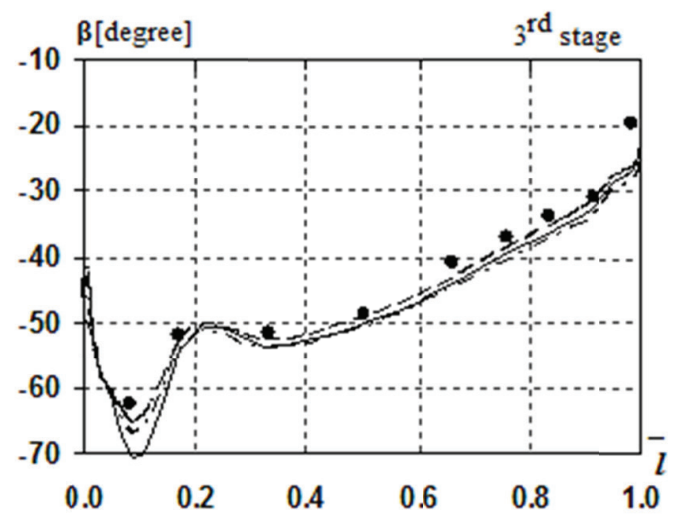


e)

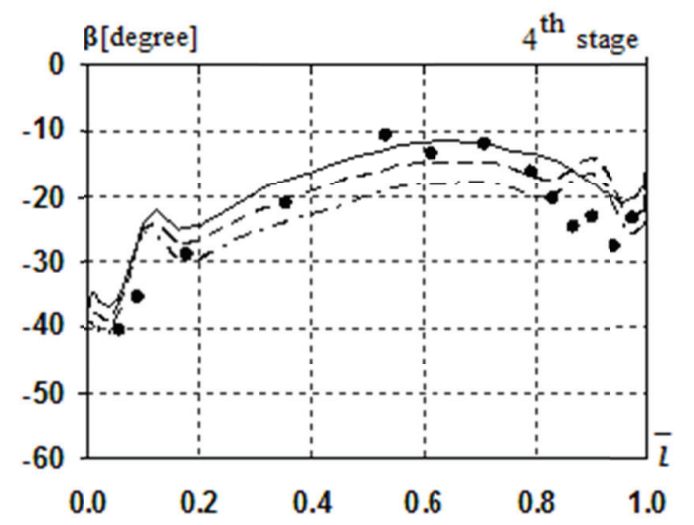

f)

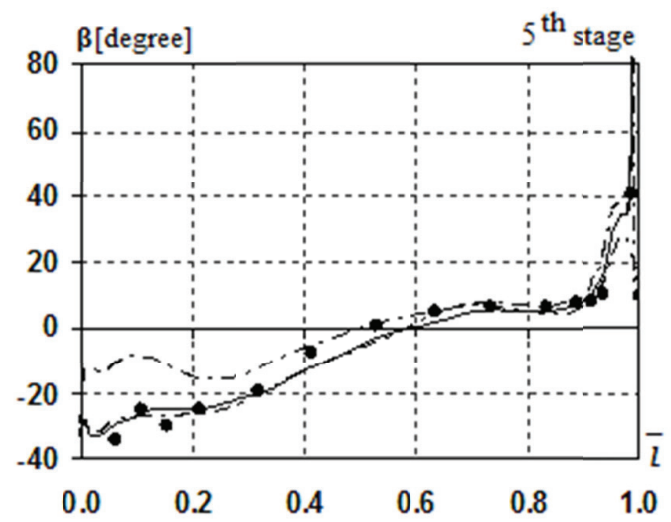

Fig. 4. Pitch angle $(a, b, c)$ and swirl angle $(d, e, f)$ distribution along the channel height in stage 3, 4 and 5;

-. experiment, ----- calculation 1,-- -- -- calculation 2, ------ calculation 3

\section{CONCLUSIONS}

The paper presents an approach to approximate equations of state for water and steam (IAPWS-95) for the calculation of three-dimensional flow in an LP steam turbine. The comparison of numerical results with experimental data is also presented. The average approximation error of evaluation of thermodynamic functions within the working area of turbine is lower than $0,01 \%$, the maximum error remains below $0,1 \%$. Test calculations of three-dimensional viscous flow in an LP steam turbine are also made using various equations of state (perfect gas, Van der Waals equation, equation of state for water and steam IAPWS-95). It is shown, that the presented method of approximation of IAPWS-95 properties provides the best fit of computational and experimental results.

\section{LITERATURE}

1. ANSYS Products, Terms \& Conditions. ANSYS, Inc. All Rights Reserved, available on: http://www.ansys.com

2. Wróblewski W., Dykas S., Gepert A., 2009, Steam condensing flow modeling in turbine channels, Int. J. Multiphase Flow; Vol. 35(6), pp. 498-506.

3. Yershov S., Rusanov A., 1996, The Application Package FlowER for the Calculation of 3D Viscous Flows Through Multi-Stage Turbomachinery. Certificate of state registration of copyright No. 77, Ukrainian Agency for Copyright and Related Rights, 19.02.1996.

4. Nashchokin V., Semyonov S., 1980, Engineering Thermodynamics and Heat Transfer, Moscow, High School.

Table 2. Integral characteristics of the investigated LP turbine

\begin{tabular}{|c|c|c|c|c|}
\hline Parameter & Experiment & Calculation 1 & Calculation 2 & Calculation 3 \\
\hline \multicolumn{5}{|c|}{ In the outlet part - stage 1} \\
\hline $\mathrm{P}, \mathrm{kPa}$ & - & 321,23 & 16,1 & 314,4 \\
\hline $\mathrm{T}, \mathrm{K}$ & - & 504,85 & 00,7 & 494,4 \\
\hline \multicolumn{5}{|c|}{ In the outlet part - stage 2} \\
\hline $\mathrm{P}, \mathrm{kPa}$ & - & 190,31 & 86,7 & 182,8 \\
\hline $\mathrm{T}, \mathrm{K}$ & - & 471,24 & 64,2 & 443,0 \\
\hline $\mathrm{T}, \mathrm{K}$ & 371,2 & 421,04 & 11,2 & 367,4 \\
\hline $\mathrm{I}, \mathrm{kJ} / \mathrm{kg}$ & 2647 & 2690 & 2643 & 2646 \\
\hline $\mathrm{G}, \mathrm{kg} / \mathrm{c}$ & 107,9 & 108,31 & 08,7 & 107,6 \\
\hline \multicolumn{5}{|c|}{ In the outlet part - stage 4} \\
\hline $\mathrm{P}, \mathrm{kPa}$ & 34,9 & 35,86 & 35,3 & 35,32 \\
\hline \multicolumn{5}{|c|}{ In the outlet part - stage 5} \\
\hline $\mathrm{P}, \mathrm{kPa}$ & 8,3 & 8,24 & 8,22 & 8,23 \\
\hline $\mathrm{T}, \mathrm{K}$ & 314,8 & 320,53 & 20,6 & 313,7 \\
\hline $\mathrm{I}, \mathrm{kJ} / \mathrm{kg}$ & 2350 & 2390 & 2387 & 2370 \\
\hline $\mathrm{G}, \mathrm{kg} / \mathrm{c}$ & 96,0 & 100,1 & 91,7 & 95,1 \\
\hline
\end{tabular}


5. IAPWS-95, Revised Release on the IAPWS Formulation 1995 for the Thermodynamic Properties of Ordinary Water Substance for General and Scientific Use, available on: http://www.iapws.org

6. IAPWS-IF97, Revised Release on the IAPWS Formulation 1997 for the Thermodynamic Properties of Water and Steam, available on: http://www.iapws.org

7. Yershov S., Rusanov A., 2002, Numerical simulation of three-dimensional viscous flows in turbomachines for imperfect gas, Part 1. Problem Statement. Probl. Engineering, Vol. 5, No. 4, pp. 18-25.

8. Menter F., 1994, Two-equation eddy viscosity turbulence models for engineering applications. AIAA J., Vol. 32, No 11., pp. 1299-1310.

9. Yershov S., 1994, Quasi ENO scheme of high accuracy for Euler and Navier+Stokes equations, Math. Modeling, Vol. 6, No. 11, p. 58-64.

10. Sommerfeld A., 1964, Thermodynamics and Statistical Mechanics (Lectures on Theoretical Physics), Academic Press.

11. Godunov S. Zabrodin A., Ivanov M., 1976, Numerical solution of multidimensional problems of gas dynamics, Moscow, Nauka.

12. Rusanov A., Yershov S., 2008, Mathematical modeling of unsteady gas-dynamic processes in flow-through parts of turbomachinery, Institute of Mechanical Engineering Problems, Kharkiv.

13. Marcinkowski S., Gardzilewicz A., Gluch J., 1999, Results of extended flow measurements in the LP part of $18 \mathrm{~K} 370$ steam turbine, Rep. Diagnostyka Maszyn, Gdańsk, No. $11 / 99$.

14. Lampart P., Rusanov A., Yershov S., Marcinkowski S., Gardzilewicz A., 2005, Validation of 3D RANS Solver With a State Equation of Thermally Perfect and Calorically Imperfect Gas on a Multi-Stage Low-Pressure Steam Turbine Flow, Journal of Fluids Engineering, Vol. 127, pp. $83-93$.

\section{CONTACT WITH THE AUTHOR}

Piotr Lampart

The Szewalski Institute of Fluid Flow Machinery Polish Academy of Sciences in Gdańsk e-mail: lampart@imp.gda.pl

\section{Poland}

